\title{
Clinical outcome of tibial spine ACL a vulsion fracture in adults treated with suture bridge fixation
}

\author{
Sujit Jos ${ }^{1}$, Rajeev Anand ${ }^{2, *}$, Joe Paul Elenjickal ${ }^{3}$ \\ ${ }^{\mathbf{1}}$ Associate Professor, Dept. of Orthopedics, SN Medical College, Ernakulam, Kerala, ${ }^{\mathbf{2}}$ Associate Professor, Dept. of Radio \\ Diagnosis, SN Medical College, Ernakulam, Kerala, ${ }^{3}$ Assistant Professor, Dept. of Orthopedics, SN Medical College, Ernakulam, \\ Kerala, India
}

\section{*Corresponding Author:}

Email: rajeevradiology@gmail.com

\begin{abstract}
Introduction: Anterior cruciate ligament avulsion from the tibia with a bony fragment of the tibial spine is an uncommon injury of the knee joint. These are predominantly seen in children and young adults. Arthroscopic reduction and fixation of the tibial avulsion fracture is a technically demanding procedure as restoring the ACL length and tension during fixation is important to ensure stability of the knee while maintaining range of motion and minimal knee laxity. The purpose of the study was to evaluate the outcome of Arthroscopic Suture bridge fixation of tibial spine anterior cruciate ligament avulsion fractures.

Materials and Methods: We followed up seven patients prospectively after Arthroscopic suture bridge fixation. The patients were assessed with clinical examination, Tegner Lysholm and IKDC (International Knee Documentation Committee) knee scores. The study group consisted of 5 males and 2 females with a mean age of 24.28 years (Range 18 years to 32 years). Mean follow-up time was 28.7 months (range 24 to 36 months). The study included 4 type II and 3 type III Meyer and McKeever fractures.

Results: At follow-up the mean Tegner lysholm score was 91.28. The best results were seen in younger patients. Younger patients had significantly better score for IKDC functional and final scores.

Conclusion: Displaced tibial spine avulsion fractures can be successfully treated in younger and older patients using arthroscopic suture bridge fixation with most patients returning back to preinjury levels of activity.
\end{abstract}

Keywords: Arthroscopy, Tibial fractures, Avulsion, Anterior cruciate ligament injuries, Fracture fixation internal.

\section{Introduction}

Tibial spine avulsion fracture of the anterior cruciate ligament are uncommon fractures and are seen in younger age group. They account for $15 \%$ of anterior cruciate ligament (ACL) injuries. They usually occur due to hyperextension of the knee when the femur rotates on the tibia and this can occur during sports like football or during road traffic accidents. The hyperextension and rotation creates tension on the ACL which extends from the lateral femoral condyle to the tibial spine, causing the avulsion fracture.

Arthroscopic reduction of the tibial spine avulsion has replaced traditional open reduction due to decreased morbidity. Early detection and reduction of Meyers and McKeever ${ }^{1}$ type II and III tibial spine fractures is important to reduce risk of non-union, knee laxity and loss of range of motion.

The results in children and adolescents are usually satisfactory, but the results in adults are less predictable. ${ }^{4}$ Some authors have reported high rates of complications such as stiffness and recurrent instability in both younger and older patients.

Berg $^{2}$ reported on 2 cases in which postoperative arthrofibrosis developed, and Montgomery et al. ${ }^{9}$ reported that 9 of 17 patients $(53 \%)$ had severe difficulty in regaining motion post-operatively. Osti et al. $^{3}$ studied 10 patients and found no extension deficit but reported a rate of laxity of $30 \%$ (3 of 10 patients) with fair or poor results. On the other hand, Zhao and Huangfu ${ }^{5}$ treated 18 patients with nonunited anterior cruciate ligament (ACL) tibial avulsion fractures with arthroscopic suture fixation and reported no case of arthrofibrosis or instability.

The purpose of the study was to evaluate the outcome of Arthroscopic Suture bridge fixation of tibial spine anterior cruciate ligament avulsion fractures in skeletally mature patients, particularly knee stability and range of motion.

\section{Materials and Methods}

We followed up 7 patients with tibial spine avulsion fractures treated with Arthroscopic suture bridge fixation, from June 2009 to July 2014 at SN Medical College, Ernakulam. The inclusion criteria were displaced tibial spine fractures (type II, III, IV) in skeletally mature patients. Tibial spine fractures were classified according to Meyer and McKeever. ${ }^{1}$ [Fig. 1]

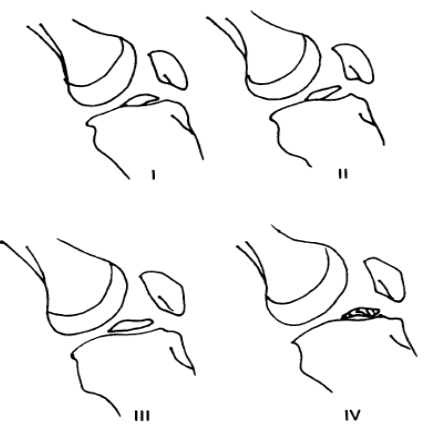

Fig. 1: Meyer and McKeever classification 
The knees were assessed preoperatively with clinical and radiological examination [Fig. 2]. Skiagrams and magnetic resonance imaging were done for all the patients to assess the extent of bony avulsion and to rule out other associated pathologies like meniscus tears or cartilage lesions.

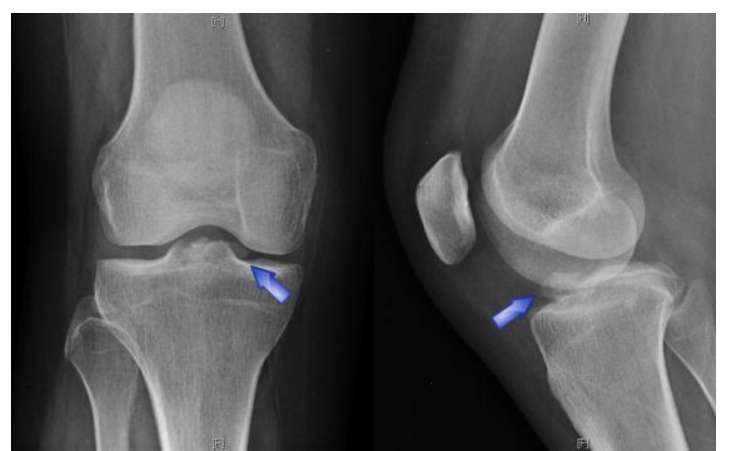

Fig. 2: Preoperative Xray showing avulsed bony fragment of tibial spine

Surgical Technique: The patients were operated by the same surgeon within 21 days of the injury. Under spinal anesthesia the patients were placed in supine position and diagnostic knee arthroscopy was performed under a tourniquet. The basic principles of suture fixation of the avulsion fractures described by Lee ${ }^{6}$ in 1937 using open techniques were followed. Arthroscopy pump was used to control bleeding and improve visualization. After evacuation of the hematoma the joint was thoroughly inspected for associated injuries. Meniscus and cartilage issues were addressed appropriately before tibial spine fracture reduction was done. The crater on tibia was freed of any soft tis sue and fracture fragments. The tibial spine avulsion fracture fragment was reduced by gentle manipulation. Any intervening soft tissue was debrided. [Fig. 3]

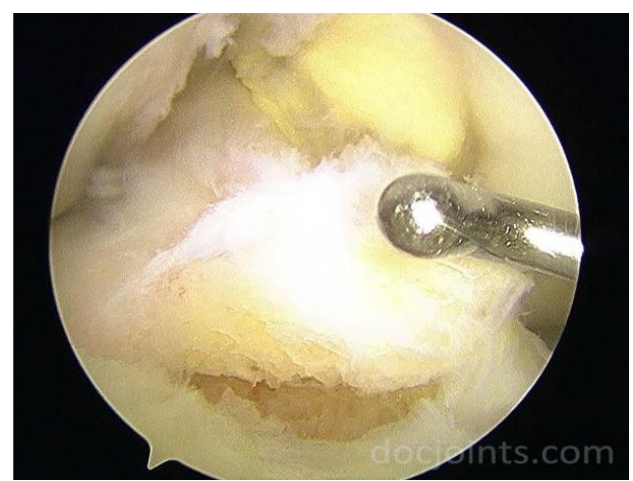

Fig. 3: Tibial spine avulsed fragment

Acupass suture shuttle (Smith \& Nephew) was used through the anteromedial portal to pierce the fibres of the ACL at the junction of anterior third and middle third. A suture grasper was used to retrieve the loop of the Acupass through the anteromedial portal. No-2 Fibre wire was passed using the suture loop of the
Acupass suture shuttle. On more similar fibre wire was passed through the fibres of the ACL at the junction middle and posterior thirds of its substance. The ends of the suture limbs were tagged with a hemostat for future identification.

A $3 \mathrm{~cm}$ longitudinal incision was made over the proximal anteromedial tibia. An ACL drill guide was used to drill two holes of $4 \mathrm{~mm}$ on either side of the tibial spine avulsion fracture [Fig. 4]. A bony bridge of at least $2 \mathrm{~cm}$ was ensured between the tunnels. The intra-articular exits of the tunnels were positioned at the anteromedial and anterolateral margins of the fracture site, respectively. The medial and lateral limbs of each of the fibre wire sutures were brought out of the medial and lateral bone tunnels respectively, using a straight spinal needle with a 2-0 PDS loop loaded in it brought retrograde through the bone tunnels in tibia.

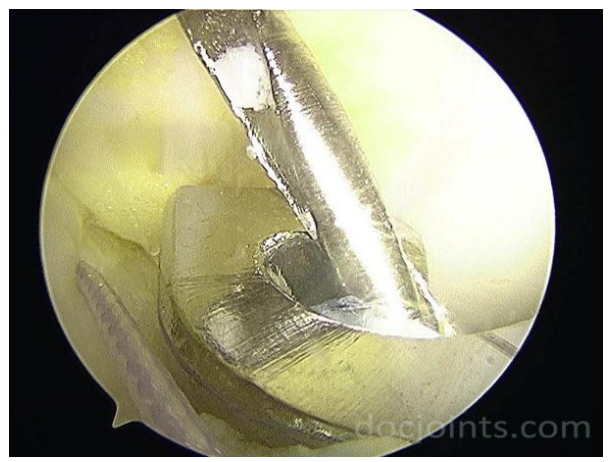

Fig. 4: Beath-pin being drilled on the side of the tibial spine fragment to pass the fibre-wire

The sutures were then tied over the bony bride with the knee in 30 degree of flexion. The fragment was held reduced using the probe or blunt trocar, while the sutures were tied in tension. The stability of the fragment was re-examined with a probe, checking the reduction and stability of the fragment throughout knee flexion and extension. ACL tension was confirmed with the probe [Fig. 5].

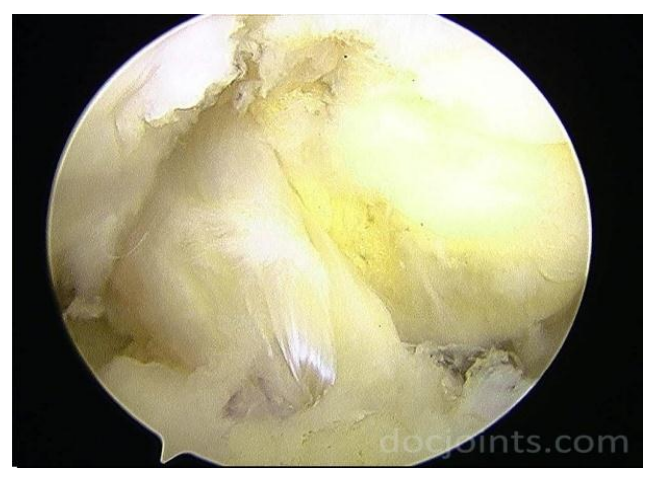

Fig. 5: Adequate tension in the anterior cruciate ligament after fixing the avulsed bony fragment

Post-operative radiographs were used to confirm anatomic reduction of the fragment [Fig. 6]. 


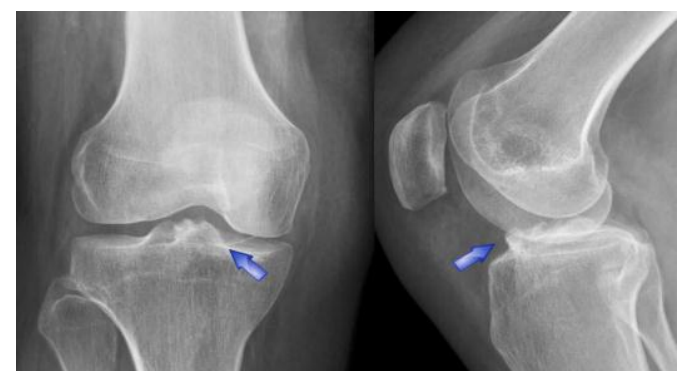

Fig. 6: Post-operative radiograph

Rehabilitation: The knee was immobilized on a long leg splint for 2 weeks. After suture removal a hinged knee brace locked in extension was used for the next 2 weeks. Patellar mobilization exercises and isometric quadriceps exercises were performed with the knee in the brace. Partial weight bearing was allowed with crutches for the first 4 weeks. At 4 weeks, range of motion exercises were initiated and gradually progressed to 90 degrees over the next month. Full weight bearing was allowed at 4 weeks with the brace locked in extension. The brace was removed at 8 weeks and further flexion of the knee was encouraged. Light jogging was permitted at 3 months. Return to sports was allowed at 6 months postoperatively, after knee stability, range of motion, muscle strength and proprioception were restored.

Clinical Evaluation: The patients were followed up at 6 weeks, 3 months, 6 months, 12 months and then yearly. Anteroposterior and lateral radiographs were obtained at 6 weeks and 3 months postoperatively to assess fracture union. The proximal migration of the fragment was assessed using digital radiographic measurement tools, after compensating for the magnification. At final follow-up the patients were assessed by an independent orthopedic surgeon. A comprehensive clinical examination was performed to assess the lachman, pivot-shift and anterior drawer tests. The tibial translation was graded by the examiner to quantify the stability. Range of motion was evaluated actively and passively with a goniometer. Lysholm and International Knee Documentation Committee (IKDC) knee examination form were used to evaluate knee function. Activity level before injury and at follow-up was graded by the Tegner scale. The knees were graded as normal (grade A), nearly normal (grade B), abnormal (grade C), or severely abnormal (grade D). Knee radiographs in standing anteroposterior, standing lateral, or merchant views were examined for alignment, joint space narrowing and degenerative changes.

\section{Results}

Twelve patients met the inclusion criteria. But three of them had associated tibial plateau fracture or other limb injuries and were excluded from the study. 2 patients were lost to follow-up and were excluded from the study. The final study population consisted of 5 male $(71 \%)$ and 2 female (29\%) patients. The mean age at the time of surgery was 24.28 years (range, 18 to 32 years). The cause of injury was related to sports in 4 (57\%), road traffic accident in $2(28.5 \%)$ and trivial fall in $1(14 \%)$. As per modified Meyer and McKeever classification 4 patients (57\%) were identified as type IV and 3 patients (43\%) were type III. The mean time to surgery was 3.14 days (range, $1-8$ days).

Lachman test demonstrated pathologic laxity (>grade 2) in all patients. Range of motion was found to be on average $7.4^{\circ}$ of extension (range, $0-10^{\circ}$ ) and $75.71^{\circ}$ of flexion (range, 20-120 $)$. The Lysholm or IKDC scoring was not done preoperatively as all the injuries were acute. Associated injuries were found in 4 patients (57\%). Medial meniscus tears were found in 3 patients $(42 \%)$, lateral meniscus tear in one $(14 \%)$ and medial femoral cartilage injury in one patient (14\%). All meniscus injuries were treated by partial meniscectomy and balancing and the medial femoral condyle cartilage injury was treated with radiofrequency coablation. The average follow-up time was 28.7 months (range, 24 to 36 months).

Follow-up radiographs obtained at 3 months showed that all the fractures had healed anatomically [Fig6]. The latest follow-up radiographs did not show any sign of degeneration in any of the patients.

At follow-up interview, all the patients were satisfied with the results and had good functional activity. All of then returned to preinjury function including sports. The mean preinjury Tegner Lysholm score was 100 , and at follow-up evaluation the average score was 91.28 (range, 81 to 99 ). The IKDC score showed and average preinjury value of 100 , and at follow-up evaluation the average score was 96.08 (range, 92 to 100). One patient required a second look arthroscopy for a clicking in the knee near full extension. It was found to be due to synovial thickening near the repaired tibial spine fragment and was treated by arthroscopic debridement which corrected the problem intra-operatively. His knee scores improved afterwards and returned to preinjury activity at further follow-up).

\section{Discussion}

Meyers and McKeever ${ }^{1}$ first classified these fractures into 3 types. A type I fracture exhibits minimal displacement with good bone apposition, whereas a type II fracture represents displacement of the anterior one third to one half of the avulsed fragment, which is hinged at the posterior border. A type III fracture has complete displacement and usually rotation of the fragment from the proximal tibial epiphysis. Later, Zaricznyj ${ }^{7}$ described a fourth type of tibial eminence fracture involving excessive comminution of the fracture fragment. Meyers and McKeever ${ }^{1}$ recommended closed reduction and immobilization for type I and II fractures, and open reduction and internal fixation for type III fractures. 
Since then, many techniques have been described for the treatment of tibial spine fractures. Previously, displaced type III fractures required an arthrotomy to achieve adequate reduction; however, this approach has been associated with increased operative time and length of hospitalization, as well as a second operation for hardware removal. As a result, some authors continue to favor conservative treatment for all tibial eminence fractures, while others suggest operative intervention for types II, III, and IV.1 Currently, operative techniques range from arthrotomy with screws, wires, staples, pins, or suture fixation to arthroscopically assisted internal fixation with cannulated or cortical screws, percutaneous pins, and suture fixation with and without hardware. Consequently, arthroscopic treatment of these fractures is increasing using an anterior cruciate ligament (ACL) tibial drill guide for fragment reduction with fixation to a screw and post or over a bony bridge.

Avulsion fractures of the tibial spine occur most frequently in children and adolescents and are caused most commonly by sports injuries and falls from bicycles. Most tibial spine fractures in children have a good prognosis, with recent attention directed toward surgical management of type II, III, and IV fractures. Type I fractures can be managed nonsurgically with cast immobilization for 6 to 8 weeks. Type II fractures have also been successfully treated using closed reduction and cast immobilization, but several authors believe that manipulation during the closed reduction of the fracture fragment may cause subsequent displacement. Furthermore, McClennan ${ }^{8}$ showed that the fracture "footprint" is not congruous with the femoral condyle in extension or flexion, thus preventing complete fragment reduction and full knee range of motion. He determined that the reduction is maintained by "lack" of ligament tension. Surgical treatment is warranted for type III and IV fractures after reports of increased laxity, loss of knee extension, chondromalacia, quadriceps weakness, malunion, and nonunion in patients treated nonsurgically. McClennan ${ }^{8}$ reported an average 6-year follow-up of 10 patients with type III fractures treated using both operative and nonoperative treatment. Second-look arthroscopy revealed greater than $3-\mathrm{mm}$ offset in patients treated with closed and arthroscopic reduction alone. He concluded that type III fractures that are irreducible or displaced require reduction, appropriate tensioning, internal fixation, and aggressive rehabilitation. Numerous arthroscopic and open techniques have been described for type III fractures.

\section{Conclusion}

Displaced tibial spine avulsion fractures can be treated successfully in younger and older patients using arthroscopic suture bridge fixation with most patients returning back to preinjury levels of activity. There is significantly lower rate of reoperation when compared to fixation with screws or other hardware.

Funding: No funding sources

Conflict of Interest: None declared

Ethical Approval: The study was approved by the institutional ethics committee.

\section{References}

1. Meyers MH, McKeever FM. Fracture of the intercondylar eminence of the tibia. J Bone Joint Surg Am 1959;41:209-222.

2. Berg EE. Comminuted tibial eminence anterior cruciate ligament avulsion fractures: Failure of arthroscopic treatment. Arthroscopy 1993;9:446-450.

3. Osti L, Merlo F, Bocchi L. Our experience in the arthroscopic treatment of fracture-avulsion of the tibial spine. Chir Organi Mov 1997;82:295-299.

4. Hunter RE, Willis JA: Arthroscopic fixation of Avulsion fractures of the Tibial Eminence: Technique and Outcome : Arthroscopy 2004; 20: 113-121

5. Zhao J, Huangfu X. Arthroscopic treatment of non-united anterior cruciate ligament tibial avulsion fracture with figure-of- 8 suture fixation technique. Arthroscopy 2007;23:405-410.

6. Lee HG. Avulsion fracture of the tibial attachments of the cruciate ligaments. Treatment by operative reduction. $J$ Bone Joint Surg 1937;19:460-468.

7. Zaricznyj B. Avulsion fracture of the tibial eminence: Treatment by open reduction and pinning. J Bone Joint Surg Am 1977;59:1111-1114.

8. McClennon JG. The role of arthroscopic surgery in the treatment of fractures of the intercondylar eminence of the tibia. J Bone Joint Surg Br 1982;64:477-480.

9. Montgomery KD, Cavanaugh J, Cohen S, Wickiewicz TL, Warren RF, Blevens F. Motion complications after arthroscopic repair of anterior cruciate ligament avulsion fractures in the adult. Arthroscopy 2002;18:171-176.

10. Tegner Y, Lysholm J. Rating systems in the evaluation of knee ligament injuries. Clin Orthop Rel Res 1985;198:4349. 